\title{
Comunicação
}

[Communication]

\section{Densidade de estocagem na produção de juvenis de duas espécies de lambaris em sistema de recirculação}

\author{
[Stocking density in the juvenile production of two characins species \\ in a recirculating system] \\ A. Jatobá ${ }^{1}$ e B.C. Silva ${ }^{2}$ \\ ${ }^{1}$ Instituto Federal Catarinense - Araquari, SC \\ ${ }^{2}$ Empresa de Pesquisa Agropecuária e Extensão Rural de Santa Catarina - Centro de Desenvolvimento em \\ Aquicultura e Pesca - EPAGRI-CEDAP - Florianópolis, SC
}

O gênero Astyanax representa aproximadamente cem espécies, com ampla distribuição geográfica e cujo conhecimento taxonômico é bastante confuso. Os lambaris são espécies nativas de pequeno porte e com ciclo de vida rápido, aceitam alimentação artificial com bastante facilidade e apresentam elevada produtividade em cultivo intensivo, podendo atingir uma produtividade de 100 t.ha $^{-1}$ por ano, demonstrando seu potencial à piscicultura (Garutti, 2003; Porto-Foresti et al., 2010).

Para aprimorar as técnicas do cultivo de lambaris são necessários diversos estudos. E uma das primeiras etapas consiste em determinar a densidade de estocagem ideal que deve ser adotada para cada espécie com intuito de maximizar as produtividades e reduzir risco de perdas. A densidade de estocagem pode interferir no ganho em peso, taxa de crescimento, eficiência alimentar e sobrevivência dos peixes (Jobling, 1994), além de alterar o comportamento dos animais (Martins et al., 2012). A densidade ótima de estocagem, para piscicultor, depende do investimento por área de produção, sistema de cultivo, custo dos juvenis e/ou alevinos, taxa de crescimento, preço e tamanho para comercialização, entre outros fatores, sendo todos esses fatores determinantes para maximizar a produção de peixes (Merino et al., 2007).

O objetivo deste trabalho foi avaliar a densidade de estocagem na produção de alevinos de duas espécies de lambaris (Astyanax bimaculatus e A. scabripinnis), em um sistema de recirculação.

Foram realizados dois experimentos no Laboratório de Aquicultura do IFCatarinense, campus Araquari. Ambos foram aprovados pelo comitê de ética de uso animal do próprio campus ( ${ }^{\circ}$ de protocolo 0005/2013).

O primeiro experimento foi realizado com 1.080 lambaris do rabo prata (A. scabripinnis), com peso médio de $0,58 \pm 0,03 \mathrm{~g}$, entre os meses de abril a junho de 2013 (70 dias). Já o segundo experimento foi realizado com 1.080 lambaris do rabo amarelo (A. bimaculatus), com peso médio de $0,25 \pm 0,05 \mathrm{~g}$, entre os meses de abril a junho de 2014 (56 dias). Todos os lambaris foram oriundos de reproduções naturais realizadas no Laboratório de Aquicultura, de acordo com as recomendações de Garutti (2003).

Para ambas as espécies, os lambaris foram coletados e transferidos para caixas de polietileno (36L úteis) equipadas com sistema de recirculação ( $50 \%$ do volume por hora) e filtro dry wet, para permitir a manutenção da qualidade de água, garantindo a homogeneidade nos parâmetros de qualidade de água de todos os tanques.

Doze aquários foram divididos em quatro tratamentos $(9,27,81$ e 243 peixes por aquário ou $0,25,0,75,2,25$ e 6,75 peixes. $\left.L^{-1}\right)$, em triplicata.

Recebido em 24 de outubro de 2014

Aceito em 11 de junho de 2015

E-mail: adolfo.jatoba@ifc-araquari.edu.br 
Os animais foram alimentados, ad libitum, quatro vezes ao dia $(8,11,13$ e 16 horas); foram utilizadas ração em pó (níveis de garantia segundo o fabricante: mín. proteína bruta 55\%, máx. umidade $10 \%$, mín. gordura $7 \%$, máx. fibra bruta 2,8\%, mín. fósforo 1,5\%, Guabi Brasil) até todas as unidades experimentais atingirem média superior a $1,5 \mathrm{~g}$, e ração extrusada $1 \mathrm{~mm}$ (níveis de garantia segundo o fabricante: mín. proteína bruta $45 \%$, máx. umidade $10 \%$, mín. gordura $8 \%$, máx. fibra bruta $2,8 \%$, mín. fósforo $1,5 \%$, Guabi Brasil) até o final do experimento. O oxigênio dissolvido e a temperatura foram monitorados às 9 e 15 horas, diariamente, com auxílio de um oxímetro (YSI PRO2030), pH, com auxílio de um pHmetro (Waterproof Tester, HANNA), amônia total e nitrato de acordo com a metodologia descrita no APHA (2005), semanalmente.

Quinzenalmente foram realizadas biometrias para estimar o ganho de peso e crescimento dos lambaris. Ao final dos experimentos avaliaramse o peso final, ganho em peso semanal ([média final - média inicial $] \div$ semanas de cultivo), sobrevivência ([população final $\div$ população inicial] $x$ 100), eficiência alimentar aparente ([biomassa final - biomassa inicial] $\div$ ração ofertada) e produtividade aparente ([biomassa final - biomassa inicial $] \div$ volume útil dos aquários).

Os dados foram previamente submetidos à análise de Bartlett para verificar a homogeneidade de variância dos dados; os dados de produtividade foram transformados em raiz $(\mathrm{x}+0,5)$. Todos os dados foram avaliados por meio da ANOVA e regressão linear; quando observadas diferenças significativas foi realizado o teste Tukey para separação de médias, todas as análises com um nível de 5\% de significância (Zar, 2010). Os efeitos da densidade do cultivo nas respostas zootécnicas (peso final, produtividade e eficiência alimentat) também foram avaliados mediante o uso de modelos de regressões. O ajustamento dos dados ao modelo foi verificado com base na significância $(p<0,05)$ dos coeficientes de regressão pelo teste t, no coeficiente de determinação ( $\mathrm{R} 2$ = S.Q.Reg. $\div$ S.Q.Tratamento), na soma de quadrado dos desvios e no fenômeno em estudo.

Nos parâmetros de qualidade de água, independentemente do experimento $\left(1^{\circ}\right.$ ou $\left.2^{\circ}\right)$, não foram observadas diferenças significativas entre os tratamentos (Tab. 1).

Tabela 1. Valores (médias \pm desvio padrão) dos parâmetros de qualidade da água do cultivo dos lambaris do rabo prata (Astyanax scabripinnis) e do rabo amarelo (A. bimaculatus) submetidos a diferentes densidades de estocagem

\begin{tabular}{|c|c|c|c|c|c|c|}
\hline Espécie & $\begin{array}{l}\text { Densidade } \\
\left(\text { peixes.L } L^{-1}\right)\end{array}$ & $\begin{array}{l}\mathrm{O}_{2} \text { dissolvido } \\
\left(\mathrm{mg} \cdot \mathrm{L}^{-1}\right)\end{array}$ & $\begin{array}{c}\text { Temperatura } \\
\left({ }^{\circ} \mathrm{C}\right)\end{array}$ & $\mathrm{pH}$ & $\begin{array}{c}\text { Amônia } \\
\text { Total } \\
\left(\mathrm{mg}^{\left.-L^{-1}\right)}\right.\end{array}$ & $\begin{array}{l}\text { Nitrato } \\
\left(\mathrm{mg} . \mathrm{L}^{-1}\right)\end{array}$ \\
\hline \multirow{4}{*}{ A. scabripinnis } & 0,25 & $4,79 \pm 1,62$ & $23,21 \pm 1,79$ & $7,09 \pm 0,11$ & $0,11 \pm 0,10$ & $0,07 \pm 0,08$ \\
\hline & 0,75 & $4,53 \pm 1,58$ & $23,39 \pm 1,61$ & $7,14 \pm 0,14$ & $0,09 \pm 0,08$ & $0,07 \pm 0,09$ \\
\hline & 2,25 & $3,61 \pm 2,67$ & $23,32 \pm 2,33$ & $7,08 \pm 0,18$ & $0,13 \pm 0,08$ & $0,10 \pm 0,10$ \\
\hline & 6,75 & $3,14 \pm 1,74$ & $23,71 \pm 1,73$ & $6,94 \pm 0,16$ & $0,15 \pm 0,11$ & $0,11 \pm 0,12$ \\
\hline \multirow{4}{*}{ A. bimaculatus } & 0,25 & $5,79 \pm 2,04$ & $23,33 \pm 2,71$ & $7,41 \pm 0,19$ & $0,12 \pm 0,07$ & $0,08 \pm 0,08$ \\
\hline & 0,75 & $6,13 \pm 2,32$ & $23,28 \pm 2,61$ & $7,38 \pm 0,18$ & $0,10 \pm 0,09$ & $0,09 \pm 0,11$ \\
\hline & 2,25 & $5,38 \pm 1,92$ & $23,33 \pm 2,71$ & $7,19 \pm 0,23$ & $0,14 \pm 0,11$ & $0,12 \pm 0,12$ \\
\hline & 6,75 & $4,84 \pm 2,06$ & $23,39 \pm 2,83$ & $7,23 \pm 0,16$ & $0,13 \pm 0,09$ & $0,10 \pm 0,12$ \\
\hline
\end{tabular}

A concentração de amônia não ionizada (tóxica), nos dois experimentos, foi inferior a $0,002 \mathrm{mg} . \mathrm{L}^{-}$ 1 , para todas as unidades experimentais, estando em valores abaixo das concentrações tóxicas para peixes neotropicais (Martinez et al., 2006). A presença do nitrato no sistema comprova a eficiência do filtro dry wet, pois é o produto final do processo de nitrificação (Arana, 2010). Apesar de não serem observadas diferenças significativas na disponibilidade de oxigênio dissolvido, para ambas as espécies cultivadas nas densidades 6,75 peixes. $L^{-1}$ e o lambari do rabo prata a 2,25 peixes. $\mathrm{L}^{-1}$, foram registrados valores abaixo de 4,0 mg. $\mathrm{L}^{-1}$, indicado como ideal por Porto-Foresti et al. (2010) para o cultivo do lambari, podendo ser um fator limitante para o desenvolvimento dos peixes nessas densidades, além das altas densidades. Brandão et al. (2005) 
observaram efeito semelhante no oxigênio dissolvido ao registrarem menores valores de oxigênio dissolvido nos tratamentos com maiores densidades de estocagem para matrinxã (Brycon amazonicus).

A sobrevivência não foi influenciada pelas densidades de estocagem, para ambas as espécies
(Tab. 2); fato este já observado para matrinxã (Brandão et al., 2005) pirarucus (De Oliveira et al., 2012) e jundiás (Martinelli et al., 2013). Em todos os trabalhos, as densidades de estocagem não interferiram nos parâmetros de qualidade de água; dessa forma, não comprometeram a capacidade de suporte dos sistemas de produção avaliados.

Tabela 2. Médias finais \pm desvio padrão dos índices zootécnicos dos lambaris do rabo prata (Astyanax scabripinnis) e do rabo amarelo (A. bimaculatus) submetidos a diferentes densidades de estocagem

\begin{tabular}{|c|c|c|c|c|c|}
\hline \multirow{2}{*}{ Espécie } & \multirow{2}{*}{ Índice Zootécnico } & \multicolumn{4}{|c|}{ Densidade de peixes (peixes.L-1) } \\
\hline & & 0,25 & 0,75 & 2,25 & 6,75 \\
\hline \multirow{7}{*}{ A. scabripinnis } & Biomassa inicial (g) & $5,26 \pm 0,04 a$ & $15,07 \pm 0,82 b$ & $48,64 \pm 0,97 \mathrm{c}$ & $140,94 \pm 1,18 \mathrm{~d}$ \\
\hline & Biomassa final $(\mathrm{g})$ & $24,18 \pm 1,63 a$ & $81,07 \pm 6,83 b$ & $210,83 \pm 42,98 \mathrm{c}$ & $502,66 \pm 55,07 d$ \\
\hline & Média final (g) & $2,80 \pm 0,22 \mathrm{ab}$ & $3,29 \pm 0,09 b$ & $2,68 \pm 0,47 \mathrm{ab}$ & $2,32 \pm 0,16 a$ \\
\hline & GPS (g.semana $\left.{ }^{-1}\right)$ & $0,22 \pm 0,02 \mathrm{ab}$ & $0,27 \pm 0,03 b$ & $0,21 \pm 0,05 \mathrm{ab}$ & $0,17 \pm 0,02 \mathrm{a}$ \\
\hline & Sobrevivência (\%) & $93,3 \pm 11,6$ & $91,4 \pm 7,7$ & $96,7 \pm 3,1$ & $89,3 \pm 10,7$ \\
\hline & Eficiência Alimentar & $0,21 \pm 0,07 \mathrm{a}$ & $0,43 \pm 0,15 \mathrm{a}$ & $0,61 \pm 0,02 b$ & $0,64 \pm 0,0 b$ \\
\hline & $\begin{array}{l}\text { Produtividade (kg.m }{ }^{-} \\
\left.{ }^{3}\right)\end{array}$ & $0,67 \pm 0,05 a$ & $2,25 \pm 0,19 a$ & $5,86 \pm 1,19 b$ & $13,96 \pm 1,53 \mathrm{c}$ \\
\hline \multirow{7}{*}{ A. bimaculatus } & Biomassa inicial (g) & $1,71 \pm 0,38 \mathrm{a}$ & $5,13 \pm, 91 b$ & $15,39 \pm 1,95 \mathrm{c}$ & $46,17 \pm 6,02 \mathrm{~d}$ \\
\hline & Biomassa final (g) & $30,66 \pm 5,12 \mathrm{a}$ & $73,56 \pm 9,80 b$ & $172,79 \pm 51,81 \mathrm{c}$ & $530,66 \pm 65,40 d$ \\
\hline & Média final (g) & $4,02 \pm 0,73 \mathrm{ab}$ & $4,49 \pm 1,26 b$ & $3,34 \pm 0,91 \mathrm{ab}$ & $2,62 \pm 0,17 \mathrm{a}$ \\
\hline & GPS (g.semana $\left.{ }^{-1}\right)$ & $0,55 \pm 0,10 \mathrm{ab}$ & $0,61 \pm 0,18 b$ & $0,45 \pm 0,13 \mathrm{ab}$ & $0,35 \pm 0,02 \mathrm{a}$ \\
\hline & Sobrevivência (\%) & $85,2 \pm 6,4$ & $78,3 \pm 11,8$ & $77,5 \pm 7,8$ & $82,9 \pm 12,1$ \\
\hline & Eficiência Alimentar & $0,55 \pm 0,05 \mathrm{a}$ & $0,64 \pm 0,17 \mathrm{ab}$ & $0,69 \pm 0,16 \mathrm{ab}$ & $0,91 \pm 0,27 b$ \\
\hline & $\begin{array}{l}\text { Produtividade }\left(\mathrm{kg} \cdot \mathrm{m}^{-}\right. \\
\left.{ }^{-}\right)\end{array}$ & $0,85 \pm 0,14 \mathrm{a}$ & $2,04 \pm 0,55 a$ & $4,80 \pm 1,44 b$ & $14,74 \pm 3,12 \mathrm{c}$ \\
\hline
\end{tabular}

Dados perdidos (DP); ganho em peso semanal (GPS); diferentes letras indicam diferenças significativas $(\mathrm{P}>0,05)$ na ANOVA e no Tukey para separação de médias.

O maior peso final e ganho em peso semanal foram observados nos tratamentos com 0,75 peixes. $L^{-1}$, enquanto os menores foram observados nos peixes cultivados na densidade mais elevada $\left(6,25\right.$ peixes. $\left.L^{-1}\right)$, e os tratamentos com 0,25 e 2,25 peixes. $L^{-1}$ não deferiram dos demais, para ambas as espécies (Tab. 2).

O peso final demostrou uma relação indireta com a densidade de estocagem (Fig. 1A), o mesmo observado por Chattopadhyay et al. (2013) ao elevar a densidade de estocagem de 5 para 25 Labeo rohita por $\mathrm{m}^{2}$; Lazzari et al. (2011) ao elevar a densidade de estocagem de 4,2 para $6,4 \mathrm{~kg} \cdot \mathrm{m}^{-3}$ proporcionaram redução no peso individual do jundiá (Rhamdia quelen), o mesmo foi observado para Paralichthys californicus (Merino et al., 2007). Já Martinelli et al. (2013) não observaram diferenças no desempenho zootécnico em jundiás cultivados a densidades de estocagem de 2,8 ou $8,7 \mathrm{~kg} \cdot \mathrm{m}^{-3}$ (50 ou 150 peixes. $\mathrm{m}^{-3}$ ), entretanto o período experimental foi de 60 dias, a metade do tempo utilizado por Lazzari et al. (2011), podendo justificar os resultados divergentes.

A produtividade demostrou uma correlação positiva com a densidade de estocagem (Fig. 1B), as maiores foram observadas nas densidades de estocagem de 6,25 peixes. $L^{-1}$ e as menores nos tratamentos com 0,25 e 0,75 peixes. $\mathrm{L}^{-1}$, enquanto o com 2,25 peixes. $\mathrm{L}^{-1}$ divergiu de todos, para ambas as espécies (Tab. 2). Estes resultados corroboraram outros autores (Brandão et al., 2005; Merino et al., 2007; Lazzari et al., 2011; De Oliveira et al., 2012), que relacionam positivamente o aumento da densidade de estocagem com o incremento da produtividade. 


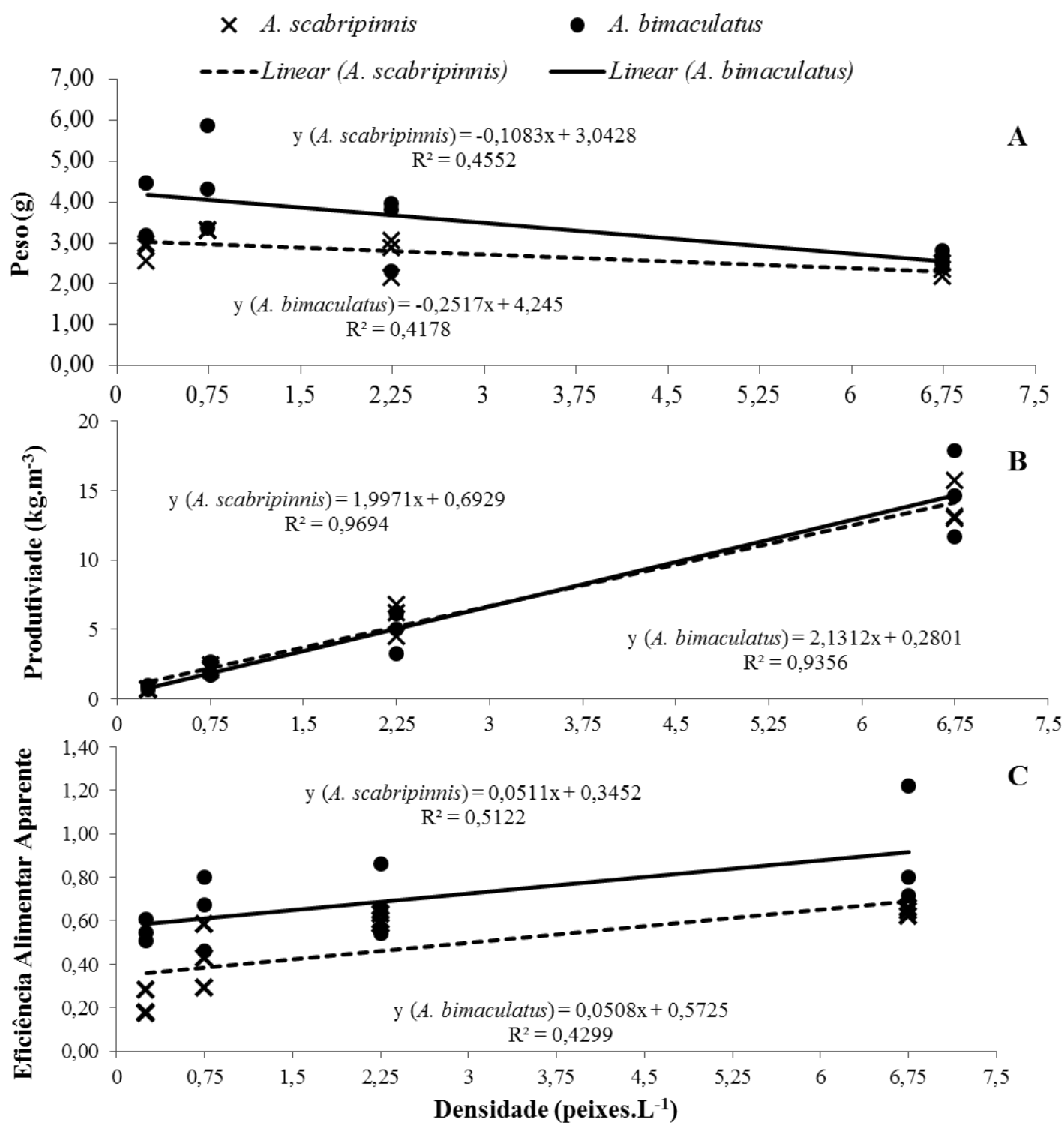

Figura 1. Regressão linear do peso final (A), produtividade (B) e eficiência alimentar aparente (C) do rabo prata (Astyanax scabripinnis) e do rabo amarelo (A. bimaculatus) submetidos a diferentes densidades de estocagem.

Vilela e Hayashi (2001) avaliaram a densidade de estocagem para juvenis de lambari (A. bimaculatus) cultivados em tanque-rede; mesmo utilizando 0,124 peixe. $\mathrm{L}^{-1}$ como maior densidade de estocagem (seis vezes menor que 0,75 peixe. $\left.L^{-1}\right)$, observaram que a menor densidade de estocagem utilizada $\left(0,031\right.$ peixes. $\left.L^{-1}\right)$ resultou em indivíduos maiores, enquanto a maior densidade $\left(0,124\right.$ peixe. $\left.\mathrm{L}^{-1}\right)$ produziu a maior biomassa. Esses resultados corroboraram as correlações obtidas neste trabalho para peso final (Fig. 1A) e produtividade (Fig. 1B), demonstrando que, independentemente do sistema de cultivo e densidades avaliadas, os lambaris apresentam a mesma correlação para densidade de estocagem e estas duas variáveis zootécnicas (ganho em peso produtividade).

A eficiência alimentar aparente (EAA) foi maior nas densidades de estocagem de 6,25 peixes. $L^{-1}$ 
em relação a 0,25 peixes. $L^{-1}$, enquanto 0,75 e 2,25 peixes. $\mathrm{L}^{-1}$ não divergiram dos demais tratamentos, para ambas as espécies. Esse efeito foi observado em bijupirás (Rachycentron canadum) submetidos a três diferentes densidades (Webb Jr. et al., 2007), assim como para Labeo rohita submetidos a seis diferentes densidades de estocagem (Chattopadhyay et al., 2013); em ambos os trabalhos, o incremento da eficiência alimentar aparente nas densidades de estocagem mais elevadas foi justificado pela maior competição por alimento, espaço e oxigênio. Para ambas as espécies de lambaris avaliados nestes estudos foram observadas correlações positivas entre $\mathrm{o}$ aumento da densidade de estocagem e EAA (Fig. 1C); além da maior competição entre os peixes, destaca-se o comportamento dessas espécies de formarem cardume em densidades elevadas. A observação do comportamento dos peixes é um indicativo sobre a qualidade do ambiente (Martins et al. 2012); nos tratamentos com menor densidade os lambaris apresentaram um comportamento mais estático e agressivo, diferente do habitualmente observado em ensaios anteriores.

O lambari do rabo amarelo apresentou maior média final em comparação com o lambari do rabo prata, esse resultado foi obtido em um menor número de dias de cultivo (56 e 70, respectivamente), além de iniciar os experimentos com menor peso inicial. A menor sobrevivência do lambari do rabo amarelo $(81,0 \%)$ em relação aos lambaris do rabo prata $(92,7 \%)$ pode ser justificada pela idade inicial dos peixes. $\mathrm{O}$ trabalho realizado com os lambaris do rabo prata utilizou peixes três vezes maiores que os utilizados no experimento com os lambaris do rabo amarelo, sendo estes mais resistentes aos manejos experimentais, como as biometrias.

Apesar de não comparar estatisticamente as duas espécies, de acordo com as observações, os autores acreditam que o lambari do rabo amarelo possui maior potencial à piscicultura, pois em condições semelhantes demonstrou índices zootécnicos mais atrativos à piscicultura.

Sendo assim, conclui-se que a elevação da densidade de estocagem no cultivo de lambaris demonstra uma correlação positiva com a produtividade e eficiência alimentar aparente, sem comprometer a sobrevivência e parâmetros de qualidade de água do cultivo. Entre as densidades avaliadas, 0,75 peixes. $\mathrm{L}^{-1}$ demonstrou maior crescimento semanal e peso final, sendo indicada para o cultivo em condições semelhantes.

Palavras-chave: Astyanax bimaculatus, $A$. scabripinnis, desempenho zootécnico, produtividade

\section{ABSTRACT}

The aim of this study was to evaluate the stocking density for juveniles for two different species of characins (Astyanax bimaculatus and A. scabripinnis) in a recirculation system. An experiment was carried out with each type in which twelve tanks in each experiment were divided into four treatments $\left(0.25,0.75,2.25\right.$ and 6.75 fish. $\left.L^{-1}\right)$, in triplicate. At the end of cultures the stocking density 0.75 fish. $L^{-1}$ showed greater weekly growth and final weight, for both species, and it is recommended for cultivation under similar conditions.

Keywords: Astyanax bimaculatus, A. scabripinnis, zootechnical performance, yield

\section{AGRADECIMENTOS}

Ao Conselho Nacional de Desenvolvimento Científico e Tecnológico $(\mathrm{CNPq})$ pelas bolsas concedidas, à GUABI por fornecer as dietas, à Karen Hartmann, Meliza Uller e Adriana de Almiron pelo apoio técnico para execução da pesquisa.

\section{REFERÊNCIAS}

ARANA, L. V. Qualidade da água em aquicultura princípios e práticas. Florianópolis: UFSC, 2010. 238p.

BRANDÃO, F.R.; GOMES, L.C.; CHAGAS, E.C. et al. Densidade de estocagem de matrinxã (Brycon amazonicus) na recria em tanque-rede. Pesqui. Agropecu. Bras., v.40, p.299-303, 2005. 
CHATTOPADHYAY, D.N.; MOHAPATRA, B.C.; ADHIKARI, S. et al. Effects of stocking density of Labeo rohita on survival, growth and production in cages. Aquacult. Int., v.21, p.1929, 2013.

GARUTTI, V. Piscicultura ecológica. São Paulo: Editora UNESP, 2003. 330p.

JOBLING, M. Fish bioenergetics. London: Chapman \& Hall, 1994. 294p.

LAZZARI, R.; RADÜNZ NETO, J.; CORRÊIA,V. et al. Densidade de estocagem no crescimento, composição e perfil lipídico corporal do jundiá. Cienc. Rural, v.41, p.712718,2011

MARTINELLI, S.G.; NETO, J.R.; SILVA, L.P. et al. Densidade de estocagem e frequência alimentar no cultivo de jundiá em tanques-rede. Pesqui. Agropecu. Bras., v.48, p.871-877, 2013.

MARTINEZ, C.B.R.; AZEVEDO, F.; WINKALER, E.U. Toxicidade e efeitos da amônia em peixes neotropicais. In: CYRINO, J.E.P.; URBINATI, E.C. Tópicos especiais em biologia aquática e aqüicultura. Jaboticabal: Sociedade Brasileira de Aquicultura e Biologia Aquática, 2006. p.81-95.

MARTINS, C.I.; GALHARDO, L.; NOBLE, C. et al.. Behavioural indicators of welfare in farmed fish. Fish Physiol. Biochem., v.38, p.17-41, 2012.
MERINO, E.G.; PIEDRAHITA, R.H CONKLIN, D.E. The effect of fish stocking density on the growth of California halibut (Paralichthys californicus) juveniles. Aquaculture, v. 265, p. 176-186, 2007.

OLIVEIRA, E.G.; PINHEIRO, A.B.; OLIVEIRA, V.Q. et al. Effects of stocking density on the performance of juvenile pirarucu (Arapaima gigas) in cages. Aquaculture, v.370, p.96-101, 2012.

PORTO-FORESTI, F.; CASTILHO-ALMEIDA, R.B.; SENHORINI, J.A.; FORESTI, F. Biologia e criação do lambari-do-rabo-amarelo (Astyanax altiparanae). In: ESPÉCIES nativas para piscicultura no Brasil. Santa Maria: UFSM, 2010. cap.4, p. 111-16.

STANDARD methods for the examination of water and wastewater. 21.ed. Washington, DC: American Public Health Association. 2005.

VILELA, C.; HAYASHI, C. Desenvolvimento de juvenis de lambari Astyanax bimaculatus (Linnaeus, 1758), sob diferentes densidades de estocagem em tanques-rede. Acta Scient., v.23, p.491-496, 2001.

WEBB JR., K.A.; HITZFELDER, G.M.; FAULK, C.K.; HOLT, G.J. Growth of juvenile cobia, Rachycentron canadum, at three diferente densities in a recirculating aquaculture system. Aquaculture, v.264, p.223-227, 2007.

ZAR, J.H. Biostatistical analysis. 5.ed. New Jersey: Pearson Prentice Hall, 2010. 994p. 